\section{Delayed forebrain syndrome due to traumatic intranasal meningoencephalocele in a cat}

\section{Theophanes Liatis ${ }^{1}$, Alberta De Stefani ${ }^{2}$, Panagiotis Mantis ${ }^{3}$, Giunio Bruto Cherubini ${ }^{1}$}

1 Neurology \& Neurosurgery, Dick White Referrals, Six Mile Bottom, United Kingdom

2 Queen Mother Hospital for Animals, Royal Veterinary College, Hatfield, United Kingdom

3 Diagnostic Imaging, Dick White Referrals, Six Mile Bottom United Kingdom

\section{OBJECTIVES}

Meningoencephalocele is a protrusion of meninges and brain through an opening in the cranium; in humans it may be congenital, traumatic, neoplastic or idiopathic, whilst in small animals mostly congenital form has been reported.

\section{METHODS}

A 3-year-old, client-owned, neutered male DSH cat was referred with one-month history of three generalized tonic-clonic seizures and intermittent left thoracic limb spontaneous knuckling. Nine months ago, the animal had been bitten on the head by a wild carnivore. Prior to the traumatic event the cat was reported to be neurologically normal.

\section{RESULTS}

On admission, physical examination was unremarkable. Neurological examination revealed left hemi-neglect syndrome, characterised by decreased postural reactions on the left thoracic and pelvic limb, and absent menace bilaterally, most likely post-ictal. Neuroanatomical localisation was right forebrain. Haematology, biochemistry and bile acid stimulation test were unremarkable. Blood serology for Toxoplasma gondii, FIV FeLV and FCoV were all negative. MRI of the head revealed protrusion of brain and meninges into the right frontal sinus, mild meningeal contrast uptake of the protruding tissue and the adjacent brain in the calvarium. CSF analysis was unremarkable. Final diagnosis was traumatic intranasal (frontoethmoidal) meningoencephalocele with late-onset forebrain syndrome due to presumptive post-traumatic encephalomalacia. The cat remained seizure-free on the four-month follow-up.

\section{STATEMENT (CONCLUSIONS)}

This is the first report of traumatic meningoencephalocele in cats. The finding in this case emphasizes the importance of a complete neurological investigation in patients with history of head trauma despite the absence of initial neurological signs. Differentials for delayed neurological signs after traumatic meningoencephalocele include encephalomalacia, recurrent meningitis with/ without CSF rhinorrhea or cerebral ischaemic infarction.

\section{Key factors that influence an owner's decision to feed their dog a senior diet}

\section{Elinor Tedds, Teresa Hollands}

School of Veterinary Medicine, University of Surrey, Guildford, United Kingdom

\section{OBJECTIVES}

To determine what influences an owner's decision to feed a senior diet. Few senior dogs in the UK are fed ageappropriate diets despite evidence that these dogs have reduced maintenance energy requirements, increased protein requirements and a reduced basal metabolic rate and that diet can be used to manage or treat age-related disease.

\section{METHODS}

A mixed methods questionnaire was distributed to dog owners via seven UK dog Facebook groups. Dogs age seven and over were classed as senior. Data analysis was carried out in Microsoft Excel.

\section{RESULTS}

$59 \%(n=214)$ of dogs in the study were classed as senior. Despite $27 \%$ of owners worrying about their dog's ageing and $84 \%$ recognising that diseases such as obesity and diabetes can be supported by diet, only $25 \%$ of senior dogs were fed senior diets. Factors influencing this decision were if the dog was fed a commercial diet, if the owner was worried about their dog's ageing, recognition that their dog's nutritional requirements change, the provision of joint support within the diet, specific ingredients in the food and if the diet was 'good quality'. $64 \%$ of owners thought glucosamine should be added to senior dog diets and $60 \%$ had spoken to their vet about nutrition of their dog.

\section{STATEMENT (CONCLUSIONS)}

Many owners speak to their vet about their dog's diet, but few feed an age-appropriate diet. This highlights a missed opportunity for veterinarians to be more proactive in discussing diet with clients and to use diet in the treatment and prevention of disease. 